\title{
Studies in the Chemistry of Some New 1,2,4-thiadiazolidine by Oxidative Cyclisation
}

\author{
Dipak T. Tayade, Rahul A. Bhagwatkar(Corresponding author) \& Radheshyam C. Panpalia \\ Department of Chemistry, S.R.R.L. Science College, MORSHI \\ Maharashtra, INDIA-444905 \\ Tel: 91-997-016-5107_E-mail: bhagwatkar83@gmail.com
}

\begin{abstract}
Recently in this laboratory a novel series of Hector's bases (1, 2, 4-thiadiazolidine) had been synthesised. 1-substituted-3-formamidinothiocarbamides (1a-f) and 1,3-bis(N-substituted-thioamido)guanidines (1g-l) were oxidatively cyclised by using aqueous bromine as oxidizing agent in chloroform medium to synthesised yet new series of Hectors bases, viz; 3-imino-5-substituted imino-1,2,4-thiadiazolidine (2a-f) and 3-substituted thioamidoimino-5-substitutedimino-1,2,4-thiadiazolidine (2g-1), respectively The oxidative cyclisation of (1a-1) was also carried out by making use of $\mathrm{H}_{2} \mathrm{O}_{2}$ in concentrated $\mathrm{HCl}$ as the oxidizing agent. The Hector's bases isolated in these reactions were characterised on the basis of conventional elemental analysis, chemical characteristics and IR and ${ }^{1} \mathrm{H}-\mathrm{NMR}$ spectral analysis.
\end{abstract}

Keywords: Hector's base, Oxidative cyclisation, 1-substituted-3-formamidino-thiocarbamide, 1, 3-bis(N-substituted thioamido)guanidines

\section{Introduction}

The first Hector's base was synthesised by Hector (1889, 1892). After Hector Hofmann and Garbial (1992), Hugershoff (1901), Kurzer (1956), Dost (1906) and Lal (1939) synthesise various series of Hector's bases and all synthesised compounds were termed and justified as Hector's bases by them. However, Sahasrabudhey (1942), Suresh (1960), Joshua (1961) investigated that all the Hector's bases which are synthesised by them are not necessarily be true Hector's bases and some were thiadiazoles and thiadiazolidines. The justification of this statement was done on the basis of gasometric studies, chemical characteristics and spectroscopic evidences (Suresh, K. S., 1959). It means that some thiadiazoles as well as thiadiazolidines are Hector's bases, but not all (For Hector's bases they must obey some typical properties (Hector D. S., 1892; Steanly, G. M., 1950; Tiwari, S. S., 1970)). The literature survey also reveals that the heterocyclic compounds having Hector's base nucleus enhance pharmaceutical, medicinal, agricultural and industrial applications. (Planka, M., 1968; Ahaluwalia, U. K., 1968; Metzger, C., 1988; Zhang Ziyi, 1969) Hence medicine containing Hector's base nucleus are now used extensively in medicinal, agricultural, pharmaceutical and biotechnological faculties. These drugs were shown to possess a diverse range of physiological activities, (Budianu, C. H., 1987; Mcguinness, 1988) plant growth promoting activity, antitumour, (Fernandes, 1986) herbical, (Farooq Saleem, 1987) antibacterial, (Farooq Saleem, 1989; Andotra, C. S., 1988) amoebicidal and antidiabetic (Rollas, S., 1984) properties. Very few work has been caring out on the synthesis of Hector's bases and their applications. As a part of research work being undertaken in the synthesis of nitrogen and sulphur containing heteroacycles and heterocycles having various application in drug chemistry, pharmaceutical, medicinal, agricultural, industrial and biotechnological sciences. Therefore, it was thought to investigate the reactions of cyanoguanidine with various isothiocyanates and also the reinvestigation of cyanoguanidine with substituted thiourea in hydrochloric acid to synthesise cyanoamidino-substituted-thiocarbamides (1a-f) and 1,3-diformamidino-thiocarbamide (2a)/N-subistituted-formamidino(formamidino)thiocarbamide (2b-e) respectively further more the oxidative cyclisation of cyanoamidino-substituted thiocarbamides and 1,3-diformamidino-thiocarbamide (2a)/N-subistituted-formamidino(formamidino)thiocarbamide with aqueous bromine in chloroform and also with $\mathrm{H}_{2} \mathrm{O}_{2}$ in concentrated hydrochloric acid gave a novel series of Hector's bases (3a-f and 4a-e) which are hitherto unknown. The present work describes suitable and somewhat direct method for the synthesis of Hector's bases. When the oxidation was carried out with $\mathrm{H}_{2} \mathrm{O}_{2}$, the yield was comparatively poor and the greater time span required for the completion of reaction. 


\section{Materials and Methods}

\subsection{1-phenyl-3-formamidinothiocarbamide (1a)}

A mixture of guanidine $(0.01 \mathrm{M})$ and phenylisothiocyanate $(0.01 \mathrm{M})$ and carbon tetrachloride $(50 \mathrm{~mL})$ was refluxed on a water bath for 2 hours. During boiling, the reaction mixture containing the suspended guanidine went into solution and after 1 hour yellowish, needle-shaped crystals gradually separated out. The reaction mixture was then again refluxed for 1 hour then filtered while hot. The new product was dried at room temperature and recrystallized from aqueous ethanol and identified as 1-phenyl-3-formamidinothiocarbamide (1a), yield $74 \%$, m.p. $130^{\circ} \mathrm{C}$. The reaction scheme was shown in Scheme-I. Similarly, other compounds (1b-f) were synthesised by above mentioned method and enlisted in Table-I.

\subsection{1,3-Bis (N-phenylthioamido)guanidine (1g)}

A similar procedure outlined in section 1.1 was used with some minor modifications, such as phenylisothiocyanate $(0.01 \mathrm{M})$ was replaced with phenylisothiocyanate $(0.02 \mathrm{M})$ Reaction yielded $67 \%$, m.p. was $149^{\circ} \mathrm{C}$. The reaction scheme was shown in Scheme-II. Similarly, other compounds (1h-1) were synthesised by above mentioned method and enlisted in Table-II.

\subsection{3-Imino-5-phenylimino-1,2,4-thiadiazolidines (2a)}

In china dish the paste of 1-phenyl-3-formamidino thiocarbamide, $(0.5 \mathrm{M})$ was prepared in chloroform. To this aqueous bromine in chloroform $(10 \%)$ was added with constant stirring. Initially the colour of bromine disappeared, the addition was continued till the colour of bromine persisted. The reaction mixture was allowed to stand for 4 hours at room conditions. A pale ivory powder separated out, which on crystallisation from ethanol, gave (2a), yield $83 \%$, m.p. $130^{\circ} \mathrm{C}$. The reaction scheme was shown in Scheme-III. Similarly, other compounds (2b-f) were synthesised by above-mentioned method and enlisted in Table - III.

\subsection{3-Phenylthiocarbamido-5-phenylimino-1,2,4-thiadiazolidines (2g)}

A similar procedure outlined in section 1.3 was used with some minor modifications, such as 1-phenyl-3-formamidino thiocarbamide was replaced with 1,3-bis-(N-phenilthioamido)guanidine Reaction yielded $79 \%$, m.p. was $195^{\circ}$ C. The reaction scheme was shown in Scheme-IV. Similarly, other compounds (2h-l) were synthesised by above-mentioned method and enlisted in Table - IV.

\section{Instrumentation}

All the chemicals used were of AnalaR grade (India make) alkyl/arylisothiocyanates were prepared according to literature method (Vogel, A. Z., 1954), melting points of all synthesised compounds were determined in open capillary and uncorrected. IR-spectra were recorded on Perkin-Elmer spectrophotometer in the range 4000-400 $\mathrm{cm}^{-1}$ in $\mathrm{KBr}$ pellets. ${ }^{1} \mathrm{H}-\mathrm{NMR}$ spectrums were recorded with TMS as internal standard using $\mathrm{CDCl}_{3}$ and DMSO- $\mathrm{d}_{6}$. The purity of the compounds was checked on silica gel-G plates by TLC.

\section{Referances}

Ahaluwalia, U. K., Dutta, U., Sharma, H. R. (1968). Ind. J. Chem., 26B, 88.

Andotra, C. S., Sharma, S. K. (1988). Pract. Nati. Acad. Sci., Ind. Sect., A 58(2), 215.

Budianu, C. H., Rusu, G., Nistor, B. (1987). Rev. Med. Chir.

Dost, K. (1906). Chem. Ber, 39, 863.

Farooq Saleem. (1987). Eur. Patent CHAPPL 87/1, 360009.

Farooq Saleem. (1989). Chem. Abstr., 110, 114893.

Fernandes, P. S., Sonar, T. M. (1986). J. Ind. Chem. Soc., 53(4), 427.

Gabriel, S., Hoffmann, A. W. (1992). Chem. Ber, 251578.

Hector D. S. (1889). Chem. Ber, 22, 1176.

Hector D. S. (1892). Chem. Ber, 25, 779.

Hector D. S. (1892). Oefvers Kong Vet. Akad., 89.

Hugershoff, A. (1901). Chem. Ber, 34, 3130.

Joshua, C. P. (1961). Tetrahedron Lett. 19, 663.

Kurzer, F. (1956). J. Chem. Soc, 2345.

Lal, R. B., Krall, H. (1939). J. Ind. Chem. Soc., 16, 31. 
Mcguinness, J. A., Minaffeli, J. A., Bell, A. R. (1988). US Patent 4775, C171-90, 408.

Metzger, C., Ludwing, E., Zeng Fuli. (1988). Gaodeng Xuexia Huazue Xuebao 9 (3), 239.

Planka, M. (1968). J. Sci. Food Agr., 19, 502.

Rollas, S. (1982). Istambul Univ. Eczacilic Fak., Mecn., 18, 3.

Rollas, S. (1984). Chem. Abstr, 101, 908429.

Sahsrabudhey, R. H., Krall, H. (1942). ibid, 19, 25.

Steanly, G. M. (1950). US Pat., 8, 252479, Chem. Abstr., 44, 59191.

Suresh, K. S. (1959). J. Sci. Res., (B.H.U.) IX (2), 94.

Suresh, K. S. (1960). J. Ind. Chem. Soc., 35, 25c.

Tiwari, S. S., Sengupta, A. K., Kumar. (1970). J. Ind. Pharma., 32, 91.

Vogel, A. Z. (1954). Text book of Practical Organic Chemistry including Qualitative Organic analysis, ELBS Landon Greek and Co., Ltd, ISBN 058244245 1, 615.

Zhang Ziyi, Yan Kexin, Helmath, H. (1969-70). S. Afr. Patent, 6805647, Chem, Abstr, 72, 7905n.

\section{Reaction Schemes}

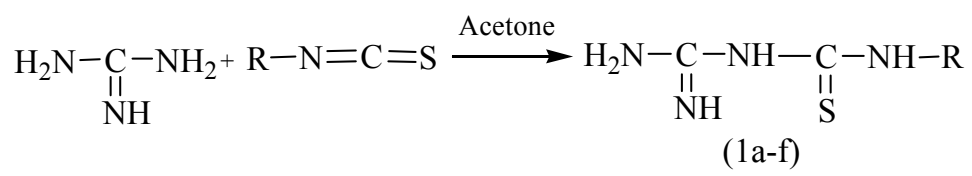

Scheme-I

$$
\begin{aligned}
& \underset{\text { NH }}{\mathrm{H}_{2} \mathrm{~N}-\underset{\mathrm{N}}{\mathrm{C}}-\mathrm{NH}_{2}}+2 \mathrm{R}-\mathrm{N}=\mathrm{C}=\mathrm{S} \underset{\mathrm{S}}{\stackrel{\text { Acetone }}{\longrightarrow}} \mathrm{R}-\mathrm{RN} \underset{\mathrm{N}}{\mathrm{I}} \underset{\mathrm{S}}{\mathrm{C}}-\mathrm{HN}-\underset{\mathrm{S}}{\mathrm{C}}-\mathrm{NH}-\mathrm{NH}-\mathrm{R} \\
& \text { (1g-1) }
\end{aligned}
$$

Scheme-II

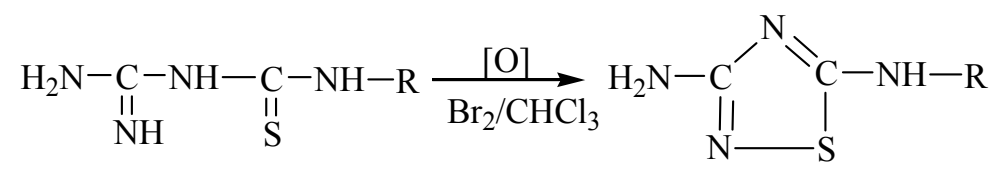

(2a-f)

Scheme-III

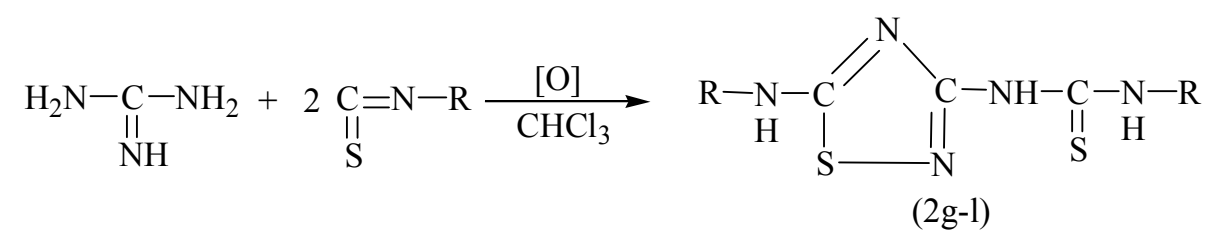

Scheme-IV 


\section{Physical data for synthesised compounds}

Table-I

\begin{tabular}{|l|l|l|l|}
\hline Compound No. & 1-substituted-3-formamidinothiocarbamide & Yield (\%) & m. p. $\left({ }^{0} \mathrm{C}\right)$ \\
\hline $1 \mathrm{~b}$ & -p-Cl-phenyl- & 84 & 142 \\
\hline 1c & -p-tolyl- & 64 & 204 \\
\hline $1 \mathrm{~d}$ & -methyl- & 72 & 189 \\
\hline $1 \mathrm{e}$ & -ethyl- & 59 & 115 \\
\hline $1 \mathrm{f}$ & -t-butyl- & 67 & 149 \\
\hline
\end{tabular}

Table-II

\begin{tabular}{|c|c|c|c|}
\hline Compound No. & 1,3-Bis(N-substituted thioamido)guanidine & Yield \% & m.p. $\left({ }^{0} \mathrm{C}\right)$ \\
\hline $1 \mathrm{~h}$ & -(N-p-Cl-phenyl thioamido)- & 82 & 194 \\
\hline $1 \mathrm{i}$ & -(N-p-tolyl thioamido)- & 72 & 189 \\
\hline $1 \mathrm{j}$ & -(N-methyl thioamido)- & 82 & 160 \\
\hline $1 \mathrm{k}$ & -(N-ethyl thioamido)- & 68 & 172 \\
\hline 11 & -(N-t-butyl thioamido)- & 62 & 169 \\
\hline
\end{tabular}

Table - III

\begin{tabular}{|l|l|l|l|}
\hline Compound No. & N-substituted-formamidino(formamidino)thiocarbamide & Yield \% & m.p. $\left({ }^{0} \mathrm{C}\right)$ \\
\hline $2 \mathrm{~b}$ & -(p-Cl-phenyl)- & 79 & 147 \\
\hline $2 \mathrm{c}$ & - (p-tolyl)- & 68 & 120 \\
\hline $2 \mathrm{~d}$ & - (methyl)- & 78 & 157 \\
\hline $2 \mathrm{e}$ & - (ethyl)- & 80 & 142 \\
\hline $2 \mathrm{f}$ & - (t-butyl)- & 63 & 110 \\
\hline
\end{tabular}

\section{Table-IV}

\begin{tabular}{|l|l|l|l|}
\hline Compound No. & N-substituted-formamidino(formamidino)thiocarbamide & Yield \% & m.p. $\left({ }^{0} \mathrm{C}\right)$ \\
\hline $2 \mathrm{~h}$ & - (p-Cl-phenyl)- & 80 & 200 \\
\hline $2 \mathrm{i}$ & - (p-tolyl)- & 83 & 182 \\
\hline $2 \mathrm{j}$ & - (methyl)- & 69 & 110 \\
\hline $2 \mathrm{k}$ & - (ethyl)- & 72 & 95 \\
\hline $2 \mathrm{l}$ & - (t-butyl)- & 54 & 124 \\
\hline
\end{tabular}

Table-V IR spectra of synthesised compounds in $\left(\mathrm{cm}^{-1}\right)$

\begin{tabular}{|l|l|l|l|l|l|l|}
\hline Compound & $v(\mathrm{~N}-\mathrm{H})$ & $v(\mathrm{C}=\mathrm{N})$ & $v(>\mathrm{C}=\mathrm{NH})$ & $v(>\mathrm{C}-\mathrm{N})$ & $v(>\mathrm{C}=\mathrm{S})$ & $v(>\mathrm{C}-\mathrm{S})$ \\
\hline$(1 \mathrm{a})$ & 3393 & 1661 & --- & --- & 1101 & 517 \\
\hline$(1 \mathrm{~g})$ & 3387 & 1666 & 1575 & 1395 & 1178 & --- \\
\hline$(2 \mathrm{a})$ & 3308 & 1660 & --- & 1332 & 1154 & --- \\
\hline$(2 \mathrm{~g})$ & 3383 & --- & 1566 & --- & 1173 & 882 \\
\hline
\end{tabular}

Table-V NMR spectra of synthesised compounds in (ppm)

\begin{tabular}{|l|l|l|l|}
\hline Compound & Ar-NH & Ar-H & N-H \\
\hline$(1 \mathrm{a})$ & $\delta 7.41-8.54$ & $\delta 6.85$ & $\delta 3.97$ \\
\hline$(1 \mathrm{~g})$ & $\delta 7.99-8.00$ & $\delta 6.90$ & $\delta 3.25-3.27$ \\
\hline$(2 \mathrm{a})$ & --- & $\delta 7.26$ & $\delta 4.02$ and 4.00 \\
\hline$(2 \mathrm{~g})$ & $\delta 7.26-7.79$ & $\delta 6.43-6.79$ & $\delta 4.02$ \\
\hline
\end{tabular}

